\title{
Shape Recovery with Concomitant Mechanical Strengthening of Amphiphilic Shape Memory Polymers in Warm Water
}

\author{
Ben Zhang, ${ }^{\dagger}$ Janae E. DeBartolo, ${ }^{\ddagger}$ and Jie Song ${ }^{*}{ }^{\dagger}$ \\ ${ }^{\dagger}$ Department of Orthopedics \& Physical Rehabilitation, University of Massachusetts Medical School, 55 Lake Avenue North, \\ Worcester, Massachusetts 01655, United States \\ ${ }^{\ddagger}$ Chemical and Materials Science Group, Advanced Photon Source, Argonne National Laboratory, 9700 Cass Avenue, Lemont, Illinois \\ 60439, United States
}

\section{Supporting Information}

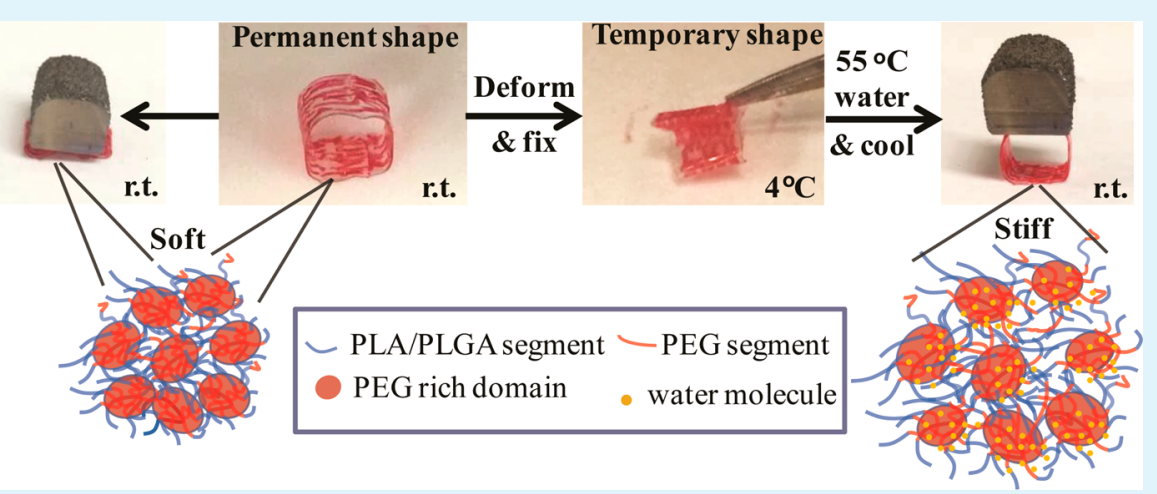

ABSTRACT: Maintaining adequate or enhancing mechanical properties of shape memory polymers (SMPs) after shape recovery in an aqueous environment are greatly desired for biomedical applications of SMPs as self-fitting tissue scaffolds or minimally invasive surgical implants. Here we report stable temporary shape fixing and facile shape recovery of biodegradable triblock amphiphilic SMPs containing a poly(ethylene glycol) (PEG) center block and flanking poly(lactic acid) or poly(lactic-coglycolic acid) blocks in warm water, accompanied by concomitant enhanced mechanical strengths. Differential scanning calorimetry (DSC), wide-angle X-ray diffraction (WXRD), and small-angle X-ray scattering (SAXS) analyses revealed that the unique stiffening of the amphiphilic SMPs upon hydration was due to hydration-driven microphase separation and PEG crystallization. We further demonstrated that the chemical composition of degradable blocks in these SMPs could be tailored to affect the persistence of hydration-induced stiffening upon subsequent dehydration. These properties combined open new horizons for these amphiphilic SMPs for smart weight-bearing in vivo applications (e.g., as self-fitting intervertebral discs). This study also provides a new material design strategy to strengthen polymers in aqueous environment in general.

KEYWORDS: amphiphilic biodegradable polymers, hydration-induced stiffening effect, minimal invasive surgery, shape memory, weight-bearing implantation

\section{INTRODUCTION}

Shape memory materials can recover from a deformed/strained temporary shape to a "memorized" permanent shape in response to stimuli such as heat, ${ }^{1-3}$ ligh, $\mathrm{t}^{4,5}$ and magnetic field. ${ }^{6}$ In thermal responsive SMPs, this is manifested by freezing and activation of polymeric chain motion below and above a transition temperature, respectively. This property is appealing for designing smart materials as minimally invasive surgical implants ${ }^{7,8}$ and self-deployable devices. ${ }^{9-11}$ Indeed, recent decades have seen great progress in constructing complex architectures ${ }^{12,13}$ and expanding actuation methods $^{14-16}$ of SMPs. For scaffold-guided tissue engineering, mechanical compliance of a biomaterial scaffold is often required for facile surgical handling/delivery while adequate mechanical strength after implantation in vivo (aqueous environment) is often desired for achieving stable fixation, particularly for weight-bearing applications. Conventional SMPs rarely address the dichotomy of these mechanical characteristics before surgical implantation/during shape programming versus after shape recovery/upon equilibration under physiological conditions. The plasticizing effect of water ${ }^{17}$ and the destruction of hydrogen bonding interactions among polymer chains cause most polymers including SMPs ${ }^{18}$ to weaken upon hydration. Thus, there is a critical need for SMPs that are capable of maintaining or strengthening their mechanical properties after shape recovery in an aqueous environment.

Received: November 5, 2016

Accepted: January 16, 2017

Published: January 26, 2017 
(a)

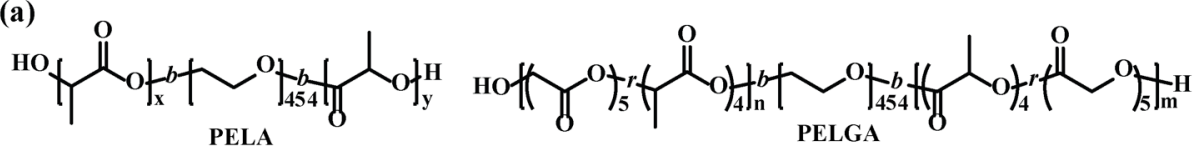

(b)
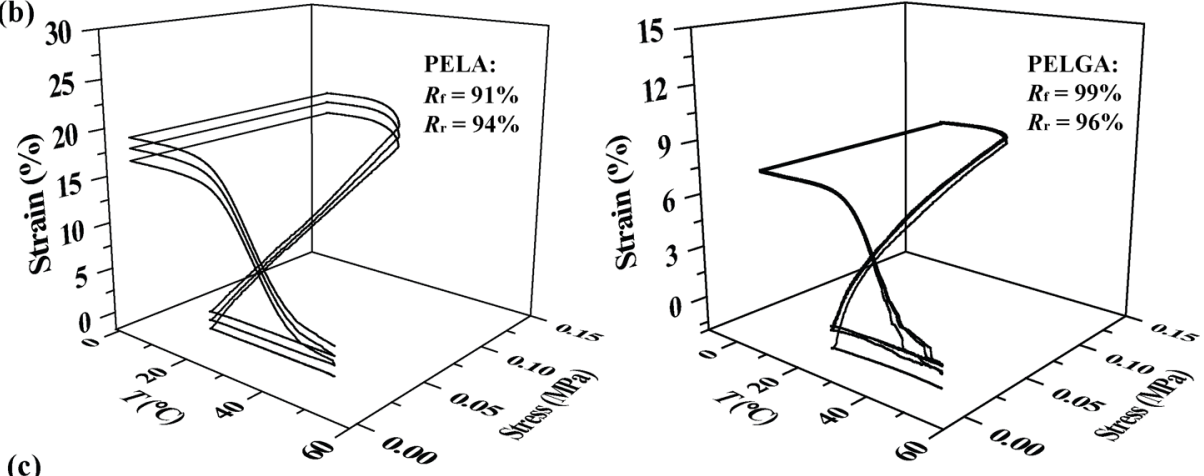

(c)
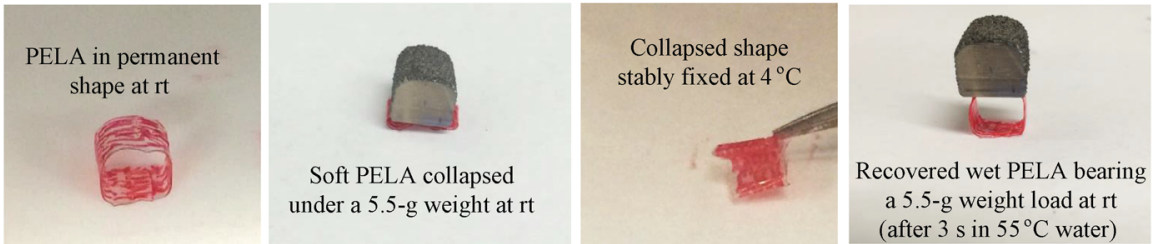

(d)
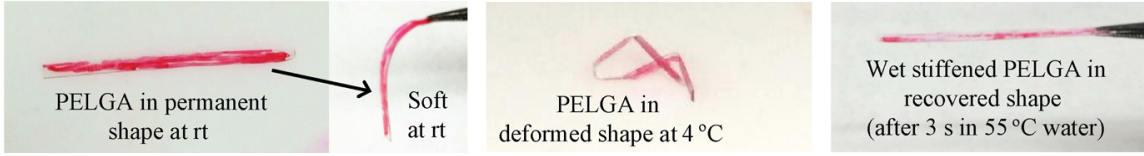

Figure 1. Thermal responsive shape memory properties of PELA and PELGA films and their facile shape recovery and stiffening in warm water. (a) Chemical structures and compositions of PELA and PELGA. $x+y=402, n+m=64$. (b) Stress-controlled cyclic thermal mechanical testing of dry PELA and PELGA films. $R_{\mathrm{f}}$ and $R_{\mathrm{r}}$ are calculated from the second cycle. (c) Facile Shape recovery of PELA in $55{ }^{\circ} \mathrm{C}$ water with concomitant mechanical strengthening. (d) Facile Shape recovery of PELGA in $55^{\circ} \mathrm{C}$ water with concomitant mechanical strengthening. Films were marked by red sharpie for easy visualization.

Some amphiphilic polymers containing PEG were recently shown to exhibit unusual hydration-induced stiffening effect, ${ }^{19,20}$ although the underlying mechanism for this phenomenon remains fully elucidated. Here we designed amphiphilic triblock poly(lactic acid)- $b$-poly(ethylene glycol)$b$-poly(lactic acid) (PLA-PEG-PLA, further abbreviated as PELA for ease of data labeling in figures) and poly(lactic-coglycolic acid)- $b$-poly(ethylene glycol)- $b$-poly(lactic-co-glycolic acid) (PLGA-PEG-PLGA, further abbreviated as PELGA for ease of data labeling) as a class of thermoplastic SMPs capable of self-stiffening upon hydration. Stress-controlled cyclic thermal mechanical testing showed that these SMPs achieved high temporary shape fixing ratio $(91-99 \%)$ at $4{ }^{\circ} \mathrm{C}$ and high shape recovery ratios $(96-99 \%)$ at $55{ }^{\circ} \mathrm{C}$. They also underwent facile shape recovery in warm water with concomitant mechanical strengthening. Using a combination of tensile mechanical testing, DSC, WXRD and SAXS, we revealed that microphase separation and crystallization differentially contributed to the stiffening effect of these amphiphilic polymer films upon hydration. In addition, we also demonstrated that the chemical composition of the degradable blocks of the amphiphilic SMPs could be used to tailor the persistence of hydration-induced stiffening upon subsequent dehydration. The safe temperature-triggered efficient shape recovery combined with stiffening of the scaffold upon equilibration in a hydrated envirnoment presents a unique opportunity of the amphiphilic SMPs as smart resorbable orthopedic implants (e.g., for restoring collapsed vertebral disc).

\section{EXPERIMENTAL SECTION}

Materials. D,L-Lactide and glycolide were purchased from SigmaAldrich (St. Louis, MO) and purified by recrystallization twice in anhydrous toluene and dried under vacuum prior to use. Poly(ethylene glycol) (PEG) (BioUltra, $20000 \mathrm{Da}$ ) was purchased from Fluka (Switzerland). All other chemicals were purchased from SigmaAldrich (St. Louis, MO) and used as received, unless noted otherwise.

Syntheses and Characterizations of PELGA and PELA. In a typical synthesis of PELGA, PEG (20000 Da, $1.0 \mathrm{~g}, 0.050 \mathrm{mmol}$ ) was heated to $100{ }^{\circ} \mathrm{C}$ in a Schlenk flask and stirred under vacuum for $2 \mathrm{~h}$ to remove residual water. The melt was cooled to room temperature before $\mathrm{Sn}(\mathrm{II})$ 2-ethylhexanoate (95\%, $3 \mathrm{mg}, 0.0074 \mathrm{mmol}$ ) in $30 \mu \mathrm{L}$ anhydrous toluene was added. After heating the mixture under vacuum at $100{ }^{\circ} \mathrm{C}$ for $15 \mathrm{~min}$ to remove toluene, the mixture was cooled to rt for the addition of D,L-lactide $(2.8 \mathrm{~g}, 33 \mathrm{mmol})$ and glycolide $(2.2 \mathrm{~g}$, $3.5 \mathrm{mmol}$ ) before the temperature was elevated to $130{ }^{\circ} \mathrm{C}$ to allow the polymerization to proceed for $5 \mathrm{~h}$ under argon with stirring. The crude polymer PELGA was dissolved in chloroform and purified by precipitation in methanol/ether mixture $(7 / 1, \mathrm{v} / \mathrm{v})$ to afford $5.0 \mathrm{~g}$ $(84 \%)$ colorless product $\left(M_{\mathrm{w}}=80119\right.$, PDI $\left.=1.7\right)$ after drying in vacuum. PELA $\left(M_{\mathrm{w}}=94761\right.$; PDI $\left.=1.7\right)$ was synthesized following prior report. $^{21}$

The molecular weights and polydispersity of PELA and PELGA were determined by gel-permeation chromatography (GPC) on a Varian Prostar HPLC system equipped with two $5 \mathrm{~mm}$ PLGel MiniMIX-D columns (Agilent, Santa Clara, CA) and a PL-ELS2100 evaporative light scattering detector (Polymer Laboratories, UK). THF 

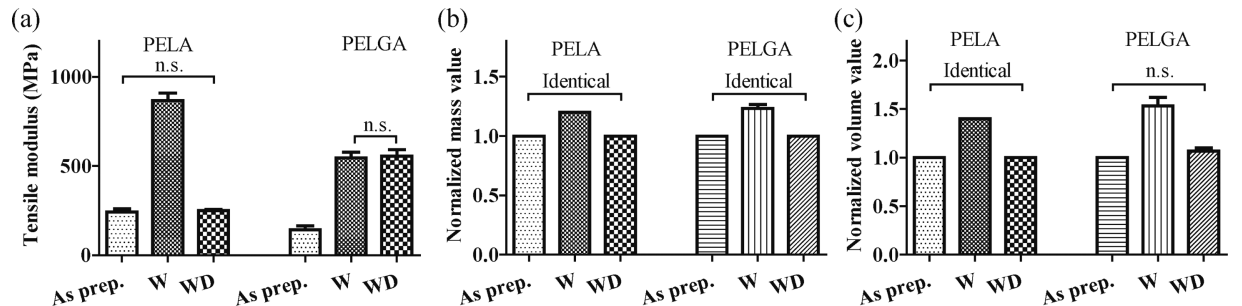

Figure 2. Room temperature (rt) hydration stiffened and swelled PELA and PELGA films while subsequent dehydration softened PELA but not PELGA. (a) Tensile moduli, (b) relative masses, and (c) relative volumes of as prepared (As prep.) cast films, wet films after $24 \mathrm{~h}$ of hydration in DI water (W), and lyophilized cast films following $24 \mathrm{~h}$ o hydration (WD) of PELA and PELGA. Specimen dimension for tensile modulus test: 5 mm $\times$ $40 \mathrm{~mm} \times \sim 0.15 \mathrm{~mm}(n=5)$. Specimen for relative masses and volume changes test: discs with a diameter of $6 \mathrm{~mm}$ and thickness $\sim 0.15 \mathrm{~mm}(n=3)$. All pairwise comparisons show statistically significant differences $(p<0.05$; student's $t$-test) unless denoted as "identical" or n.s. (not significant).

was used as an eluent at $0.3 \mathrm{~mL} / \mathrm{h}$ at $\mathrm{rt}$. Molecular weight and polydispersity calculations were calibrated with EasiVial polystyrene standards (Agilent, Santa Clara, CA). The actual glycolide to lactide incorporation ratio in PELGA was determined by ${ }^{1} \mathrm{H}$ NMR integration (Figure S1).

Preparation of Dense PELGA and PELA Films. Solvent cast films of PELGA or PELA were prepared by dissolving $\sim 1 \mathrm{~g}$ of PELGA or PELA in $\sim 3 \mathrm{~mL}$ of chloroform and poured into a Teflon mold, which was left in a ventilated fumehood overnight to allow most chloroform to evaporate. The cast film was further dried under vacuum at rt.

Cyclic Thermal Mechanical Charaterization of Shape Memory Properties. Cyclic thermal mechanical testing was carried out on a DMA Q800 equipped with a gas cooling accessory. Specimens $(5.3 \mathrm{~mm} \times 15 \mathrm{~mm} \times \sim 0.15 \mathrm{~mm})$ were equilibrated at 55 ${ }^{\circ} \mathrm{C}$ for $5 \mathrm{~min}$ and cooled to $25{ }^{\circ} \mathrm{C}$ prior to testing. After being equilibrated at $25^{\circ} \mathrm{C}$ for $5 \mathrm{~min}$, the specimens were subjected to a 0.15 $\mathrm{MPa}$ tensile stress, and cooled at $2{ }^{\circ} \mathrm{C} \mathrm{min}^{-1}$ to $4{ }^{\circ} \mathrm{C}$, while the constant stress was maintained. This yielded the elongated temporary shape under stress $\varepsilon_{1}$. After being held at $4{ }^{\circ} \mathrm{C}$ for $5 \mathrm{~min}$, the force was released to a $1 \mathrm{mN}$ force. The resulting strain was recorded as the unloaded temporary shape $\varepsilon_{\mathrm{u}}$. Shape recovery was triggered by heating the specimens at a rate of $2{ }^{\circ} \mathrm{C} \mathrm{min}{ }^{-1}$ to $55^{\circ} \mathrm{C}$ and holding at $55{ }^{\circ} \mathrm{C}$ for $5 \mathrm{~min}$. The recovered sample strain was recorded as $\varepsilon_{\mathrm{p}}$. Each specimen was subjected to three consecutive cycles of testing. The shape fixing ratio $\left(R_{\mathrm{f}}\right)$ and shape recovery ratio $\left(R_{\mathrm{r}}\right)$ were calculated from the second cycle per eqs 1 and 2 where $N$ is 2

$$
\begin{aligned}
& R_{\mathrm{f}}(N)=\frac{\varepsilon_{\mathrm{u}}(N)-\varepsilon_{\mathrm{p}}(N-1)}{\varepsilon_{\mathrm{l}}(N)-\varepsilon_{\mathrm{p}}(N-1)} \\
& R_{\mathrm{r}}(N)=\frac{\varepsilon_{\mathrm{u}}(N)-\varepsilon_{\mathrm{p}}(N)}{\varepsilon_{\mathrm{u}}(N)-\varepsilon_{\mathrm{p}}(N-1)}
\end{aligned}
$$

Tensile Modulus Determination. The tensile modulus of PELA and PELGA films $(5 \mathrm{~mm} \times 40 \mathrm{~mm} \times \sim 0.15 \mathrm{~mm})$ at $\mathrm{rt}$ were determined on a MTS Bionix 370 mechanical testing system (MTS Systems Corporation, Minneapolis, MN, USA) based on ASTM D882-97 guidelines. Specimens $(n=5)$ were loaded onto the MTS machine with an initial grip separation of $\sim 10 \mathrm{~mm}$ and subjected to a grip separation rate of $100 \mathrm{~mm} \mathrm{~min}{ }^{-1}$. The force was recorded with a 250-N load cell (Interface, Scottsdale, AZ). Tensile modulus is calculated as slope of the linear region (between $0.05 \%$ and $0.25 \%$ strain) of the recorded stress-strain curve according to standard ISO 527.

Differential Scanning Calorimetry (DSC). DSC experiments were conducted on a Q200 MDSC (TA Instruments, New Castle, DE) at a heating rate of $10^{\circ} \mathrm{C} / \mathrm{min}$ with a sample load of $\sim 1.5 \mathrm{mg}$ in an aluminum pan with the pan weight identically matched to the reference pan.

Wide-Angle X-ray Diffraction (WXRD). WXRD experiments were performed on a Rigaku XtaLAB MM007-HF including a MicroMax-007 HF Microfocus rotating anode X-ray generator,
Osmic optics and a Saturn HG CCD detector. The obtained diffraction patterns are converted to $1 \mathrm{D}$ profile by Saxsgui software.

Small-Angle X-ray Scattering (SAXS). SAXS measurements were performed at 12-ID-B at the Advanced Photon Source at Argonne National Laboratory. Monochromatic X-rays (X-ray energy is $14 \mathrm{keV}$ and wavelength $\lambda$ is $0.8856 \AA$ ) were focused on samples with a size of $0.3 \times 0.02 \mathrm{~mm}^{2}(\mathrm{HxS})$ and the scattered signals were collected on a Pilatus $2 \mathrm{M}$ detector for small angle and Pilatus $300 \mathrm{~K}$ detector for wide angle. The small angle and wide angle detectors were located about 2 and $0.3 \mathrm{~m}$ downstream of a sample, respectively. The scattering angle $2 \theta$ was calibrated with silver behenate standard and converted into scattering vector $q$ scale, where $q=4 \pi \sin \theta / \lambda$. The scattered intensity was corrected for transmittance and instrument background.

\section{RESULTS AND DISCUSSIONS}

Thermal Responsive Shape Memory Properties of PELA and PELGA and Warm-Water-Induced Shape Recovery with Concomitant Stiffening. Triblock amphiphilic polymers PELA $\left(M_{\mathrm{W}}=80119\right.$, PDI $\left.=1.7\right)$ and PELGA $\left(M_{\mathrm{W}}=94761\right.$, PDI $=1.7$; Figure 1a $)$ consisting a hydrophilic PEG $\left(M_{\mathrm{n}}=20 \mathrm{kD}\right)$ center block and two flanking hydrophobic poly(D,L-lactic acid) (PLA) blocks or hydrophobic random copolymers of D,L-lactide and glycolide (PLGA) at 4:5 ratio were synthesized by ring opening polymerization (Figure S1). Solvent-cast PELA and PELGA films (residue solvent chloroform was removed under vacuum and validated by NMR) exhibited excellent shape memory properties within a physiologically safe temperature range. Stress-controlled cyclic thermal mechanical tests of dry specimens revealed high fixation ratios $\left(R_{\mathrm{f}}\right)$ of 91 and $99 \%$ at $4{ }^{\circ} \mathrm{C}$ as well as high recovery ratios $\left(R_{\mathrm{r}}\right)$ of 94 and $96 \%$ upon equilibration at $55{ }^{\circ} \mathrm{C}$ for $5 \mathrm{~min}$ for PELA and PELGA films, respectively (Figure 1b). Interestingly, when warm water was used to trigger the shape recovery, we found that their efficient shape recovery was also accompanied by concomitant stiffening. As demonstrated in Figure 1c, a PELA thin film rolled into a permanent open ring shape was soft at $\mathrm{rt}$ and would collapse under a $5.5 \mathrm{~g}$ weight load. Upon fixing the collapsed PELA at $4{ }^{\circ} \mathrm{C}$ and then immersing in $55{ }^{\circ} \mathrm{C}$ water, it rapidly $(<3 \mathrm{~s})$ reverted to its permanent shape while significantly stiffened to readily withstand the $5.5 \mathrm{~g}$ weight load. Similarly, we also demonstrated equally efficient shape recovery and stiffening of PELGA in warm water (Figure 1d).

Differential Hydration/Dehydration-Induced Modulus, Mass and Volume Changes of PELA and PELGA Films. To better understand how hydration and dehydration differentially affect the stiffness of PELA and PELGA films, we first quantitated the tensile moduli of as-prepared, hydrated, and dehydrated films. The soft as-prepared solvent-cast PELA 
(a) SAXS
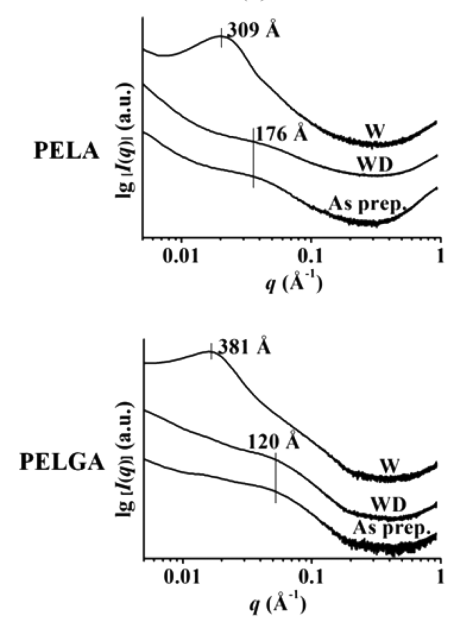

(b) DSC
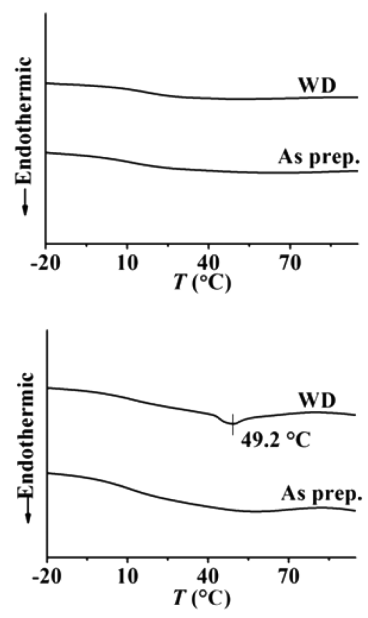

(c) WXRD
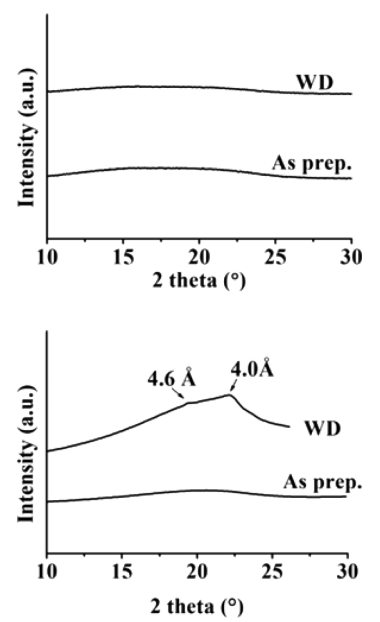

Figure 3. Structural changes of PELA (top) and PELGA (bottom) in various hydration states at rt as determined by (a) SAXS, (b) DSC (first heating cycle, $10{ }^{\circ} \mathrm{C} / \mathrm{min}$ ), and (c) WXRD. As prep.: as-prepared cast film; W: wet film after $24 \mathrm{~h}$ of hydration in DI water; WD: lyophilization-dried film following $24 \mathrm{~h}$ of hydration in DI water.

film, possessing a tensile modulus around $242 \mathrm{MPa}$ at rt, hardened upon immersion in deionized (DI) water, with its elastic tensile modulus reaching $\sim 868 \mathrm{MPa}$ after 24 -h hydration at rt (Figure 2a). It should be noted that $24 \mathrm{~h}$ hydration did not cause degradation of the polymers as their molecular weights were unchanged upon hydration (Figure S2). As water evaporated, the PELA film gradually lost its acquired stiffness and eventually reverted to its soft and compliant state (no significant difference in tensile modulus compared to the as prepared film) when fully dehydrated by lyophilization. The tensile modulus of the PELGA film also increased significantly from 142 to $546 \mathrm{MPa}$ when hydrated in DI water at rt. But different from the hydration/dehydration-induced reversible stiffening/softening of PELA, the hydration-induced stiffening of the PELGA film sustained after drying (Figure 2a).

As expected, the hydrophilic PEG center block improved the water penetration within these amphiphilic polymers, resulting in hydration-induced mass increase by $22 \%$ (Figure $2 \mathrm{~b}$ ) as well as volume increase by $41 \%$ and $53 \%$ (Figure $2 \mathrm{c}$ ) upon $24-\mathrm{h}$ equilibration in DI water for PELA and PELGA, respectively. This swelling ratio translates into $\sim 2.1$ and 2.5 water molecules per ethylene glycol repeating unit for PELA and PELGA, respectively. Both PELA and PELGA films shrank back to their initial mass and volume upon drying by lyophilization, supporting that the majority of water absorbed by the amphiphilic polymers were free water rather than structural water.

The reversible mass and volume changes of the PELA film upon hydration/dehydration are consistent with its reversible mechanical stiffening/softening behavior, suggesting that the hydration-modulated mechanics of PELA is driven by reversible structural changes. On the contrary, the reversible mass and volume changes of the PELGA film upon hydration/ dehydration did not translate into reversible mechanical stiffening/softening, suggesting that hydration may have induced PELGA to undergo structural changes that are characterized with thermodynamic stability. Such a thermodynamically stable structural change of PELGA may have been sustained upon removal of water, contributing to retained hydration-acquired stiffness upon drying.
Underlying Structural Changes of PELA and PELGA Films upon Hydration and Dehydration at rt. To reveal the reversible and thermodynamically stable structural changes of PELA and PELGA as a result of hydration/dehydration, SAXS, DSC and WXRD were carried out. A broad SAXS scattering maximum at $\sim 176 \AA(d=2 \pi / q)$ was observed with as prepared PELA film (Figure $3 \mathrm{a}$, top), indicating microphase separation of the incompatible PEG and PLA segments into hydrophobic and hydrophilic domains with an averaged domain separation of $\sim 176 \AA$. However, the intensity of this scattering was relatively weak, suggesting a relatively small electron density difference between the PLA-rich and PEG-rich domains within the polymer matrix of the as cast film. ${ }^{22,23}$ DSC (Figure $3 \mathrm{~b}$, top) and WXRD (Figure 3c, top) ruled out the formation of subnanometer sized crystal structures within the phaseseparated domains of the as prepared PELA film, with only a glass transition detected from the DSC scan while no WXRD diffraction peaks observed. Collectively, these results support a weak phase separation within the as prepared cast film of PELA that is characterized with disordered PEG and PLA chain assemblies within the respective hydrophobic and hydrophilic domains.

Enhanced phase separation was observed upon hydrating PELA at rt as indicated by a stronger and sharper SAXS scattering shifting to a lower $q$-region centered at $309 \AA$ (Figure 3a, top, $W$ curve). This larger phase-separated domain separation (309 $\AA / 176 \AA$, equivalent to 1.8 -fold increase) is consistent with the expanded macroscopic volume of PELA films upon hydration, although the degree of macroscopic volume increase appeared smaller $(\sim 1.4$ fold $)$. When the hydrated PELA film was subsequently lyophilized, its SAXS scattering maximum shifted back to $176 \AA$ along with decreased intensity (Figure 3a, top WD curve). No obvious difference was observed from the DSC and WXRD profiles before and after hydration (Figures 3b, c, top). Overall, the reversible microscopic structural changes of PELA before and after hydration/dehydration revealed by SAXS agreed with the observed reversible macroscopic volume changes and mechanical stiffening/softening. These observations support enhanced microphase separation as an underlying cause for the 
(a)

PELA

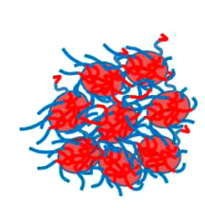

Soft/compliant

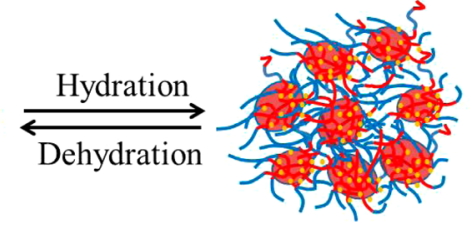

Stiff

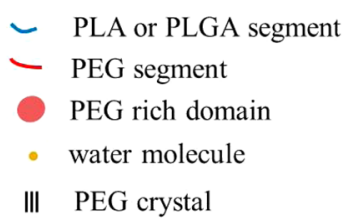

III PEG crystal

(b)

PELGA

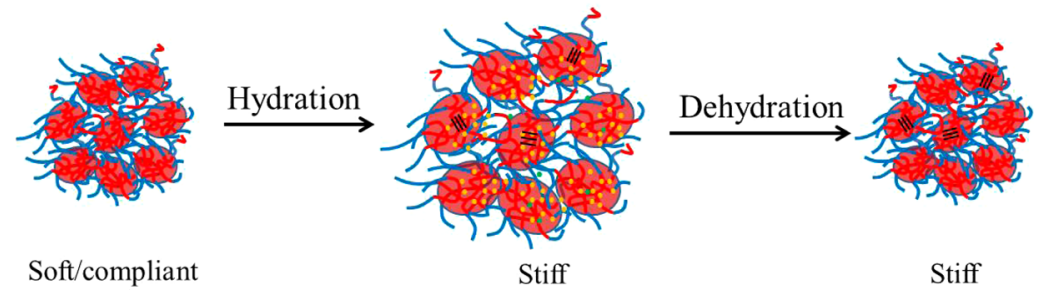

Figure 4. Proposed models for differential structural and mechanical property changes of PELA and PELGA upon rt hydration and dehydration. (a) Model of reversible phase separation upon rt hydration and dehydration of PELA. (b) Model of reversible phase separation and irreversible PEG crystallization upon rt hydration and dehydration of PELGA.

strengthened mechanical property of PELA film upon hydration at rt.

The PELGA film also underwent reversible microphase separation upon hydration/dehydration as revealed by SAXS, but its domain separations were much different from that observed with PELA. The as prepared cast film of PELGA exhibited a weak SAXS scattering at $\sim 120 \AA$, which shifted to a lower $q$-value at $381 \AA$ with enhanced intensity upon rt hydration, and returned to $120 \AA$ after drying (Figure 3a, bottom). The hydration-induced phase-separated domain expansion ratio for PELGA (381 $\AA / 120 \AA=3.2)$ is much higher than that for PELA (1.8), indicating that PELGA was more readily penetrated by water. This is consistent with the lower steric hindrance imposed by the glycolic acid compared to lactic acid side chain residues which may have translated into higher mobility of the PLGA segments in PELGA. Besides the reversible microphase separation, however, PELGA also exhibited distinctive structural changes in the subnanometer regime after hydration that was not observed in PELA as revealed by DSC and WXRD. DSC detected a broad endothermic process $\sim 40-70{ }^{\circ} \mathrm{C}$ during heating of the as prepared cast film of PELGA (Figure $3 b$, bottom, curve "As prep."), which may be attributable to the chain rearrangement of PEG segments. No detectable crystalline signal was observed from WXRD of the as prepared PELGA cast film (Figure 3c, bottom, curve "As prep"). By contrast, a more pronounced endothermic peak at $\sim 49{ }^{\circ} \mathrm{C}$ was observed by DSC scan of the PELGA cast film after hydration, implying a melt process of crystallized PEG. This speculation was supported by WXRD wherein two peaks at 4.6 and $4.0 \AA$ were observed with hydrated but subsequently lyophilized PELGA film (Figure 3c, bottom, curve WD). These diffractions are consistent with those of the crystalline PEG20k (Figure S3a) and reported PEG segments in PELGA block copolymers, ${ }^{24}$ and unlikely associated with poly(D,L-lactide-co-glycolide) segments which tend to be amorphous. ${ }^{25,26}$ Meanwhile, the wet PELGA film also gave a diffuse diffraction peak at $\sim 4.0 \AA$ (Figure S4). This hydration-facilitated PEG crystallization, in addition to microphase separation, has likely contributed to the observed hydration-induced mechanical strengthening of the PELGA film. It should be noted that the melting transition of PEG segments in PELGA, however, is $>10{ }^{\circ} \mathrm{C}$ lower than that of pure PEG20k (Figure S3b). This phenomenon is often observed in imperfect crystals containing defects/impurities with reduced thermodynamic stability. ${ }^{27}$ Thus, rt hydration likely promoted imperfect crystallization of the PEG chains in PELGA.

Models for Hydration-Induced Stiffening of PELA and PELGA. Small molecules including water often act as plasticizers to soften materials when blended into polymer. ${ }^{17}$ Here we showed that in amphiphilic block copolymers PELA and PELGA, water induced microstructural changes that translated into strengthened mechanical properties instead. As depicted in Figure 4, we suggest that the polymer chains in asprepared PELA- and PELGA-cast films adopt a "relaxed" amorphous conformation in the soft and compliant state of these materials. When water molecules penetrate through the hydrophobic segments and stabilized within PEG-rich domains, the polymer network undergoes enhanced microphase separation and is forced to expand, resulting in an increased volume and enhanced rigidity of the material. It was reported that swollen PEG-rich domains in cross-linked polymer networks could impose mechanical stress to the surrounding hydrophobic matrix, resulting in improved mechanical integrity of the material. ${ }^{28}$ Similar observation was also reported in random copolymers containing hydrophobic blocks and PEG segments. ${ }^{20}$ We postulate that besides the swelling pressure ${ }^{28}$ imposed by water molecules within the PEG-rich domains, the extension of polymer chains from their original "relaxed" state to a more taut state may have also contributed to the stiffening of the material. In the case of the PELA film, upon removal of water, the taut polymer chains shrunk back to their more relaxed state to release the internal stress, resulting in the softening of the material. Unlike PELA, PEG crystallization accompanies the microphase separation within PELGA upon rt hydration, and the more stably formed PEG crystallites within the polymer network are responsible for its sustained stiffening even upon lyophilization. Meanwhile, the more mobile hydrophobic PLGA segments in PELGA (compared to PLA segments in PELA) likely play an essential role in ensuring effective microphase separation upon $\mathrm{rt}$ hydration and permitting an sufficient yet not excessive amount of water to be localized in PEG-rich domains to facilitate the crystallization rather than dissolution of the hydrophilic PEG chains as 

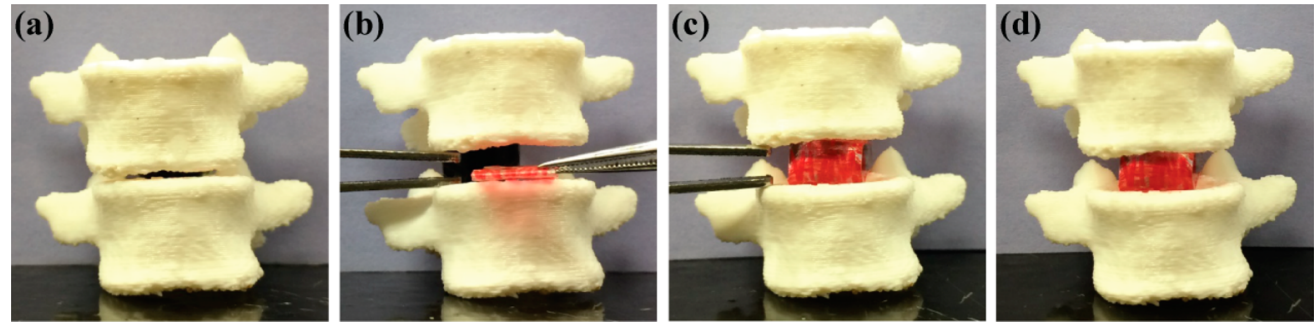

Figure 5. Demonstration of potential application of the amphiphilic SMP for restoring collpased disc space. (a) 3D printed vertebral segments showing a collapsed disc space; (b) insertion of a compressed PELGA disc (temporary shape) into the to be restored disc space; (c) shape recovery of the PELGA disc triggered by warm saline rinse to fill the disc space; (d) stiffened hydrated PELGA within the restored disc space. The PELGA disc was marked by red sharpie for easy visualization.

reported. $^{20}$ Although PEG is generally considered watersoluble, in the case of hydrated PELGA, the PEG segments adopt a higher local concentration within the swelled PEG rich domain ( $\sim 0.96 \mathrm{~g}$ of PEG in $1 \mathrm{~mL}$ of water). Comparing to the low solubility of $50 \mathrm{mg} / \mathrm{mL}$ of PEG20k in water at $25^{\circ} \mathrm{C}$, such a supra-saturated local concentration of PEG would likely lead to some degree of PEG crystallization.

The unique hydration-induced stiffening of PELA and PELGA, combined with their facile shape recovery in warm water, may open possibilities of applying these SMPs for weight-bearing biological applications. For instance, they may be applied as resorbable spine fusion cages or artificial discs for restoring collapsed vertebral disc space (Figure 5) where their delivery in a minimally invasive temporary configuration, facile shape recovery to fill the defect, and subsequent stiffening upon wetting all present unique translational advantageous.

\section{CONCLUSIONS}

In summary, we report for the first time facile shape recovery of amphiphilic triblock SMPs PELA and PELGA in warm water with concomitant strengthening of mechanical properties, and elucidated that microphase separation and PEG crystallization are responsible for their unusual hydration-induced stiffening behavior. Whereas the hardening of PELA in water at rt was primarily driven by microphase separation, hydration-acquired rigidity in PELGA film at rt resulted from both microphase separation and PEG crystallization. We also demonstrated that by adjusting the chemical composition of the degradable blocks (PLA vs PLGA blocks), we can practically regulate their mechanical properties for applications where hydration-induced stiffening needs to be maintained upon subsequent dehydration. It should be noted that an appropriate balance of hydrophobic vs hydrophilic block content is likely required to ensure that PEG crystallization rather than dissolution preferentially occurs upon hydration. This study illustrates a new strategy for the rational design of SMPs capable of strengthening their mechanical strengths upon shape recovery in an aqueous environment, broadening their utilities for weight-bearing biological applications under physiological conditions.

\section{ASSOCIATED CONTENT}

\section{S Supporting Information}

The Supporting Information is available free of charge on the ACS Publications website at DOI: 10.1021/acsami.6b14167.

${ }^{1} \mathrm{H}$ NMR spectrum of PELGA, XRD and DSC of PEG 20k, and XRD of hydrated PELGA (PDF)

\section{AUTHOR INFORMATION}

\section{Corresponding Author}

*E-mail: Jie.Song@umassmed.edu.

ORCID ${ }^{\circ}$

Jie Song: 0000-0001-7554-5564

Notes

The authors declare no competing financial interest.

\section{ACKNOWLEDGMENTS}

This work was supported by a J.R. Neff Award (Established Investigator Grant to J.S.) from the Musculoskeletal Transplant Foundation. This research used resources of the Advanced Photon Source, a U.S. Department of Energy (DOE) Office of Science User Facility operated for the DOE Office of Science by Argonne National Laboratory under Contract DE-AC02$06 \mathrm{CH} 11357$.

\section{REFERENCES}

(1) Alteheld, A.; Feng, Y. K.; Kelch, S.; Lendlein, A. Biodegradable, Amorphous Copolyester-urethane Networks Having Shape-memory Properties. Angew. Chem., Int. Ed. 2005, 44 (8), 1188-1192.

(2) Xu, J. W.; Song, J. High Performance Shape Memory Polymer Networks Based on Rigid Nanoparticle Cores. Proc. Natl. Acad. Sci. U. S. A. 2010, 107 (17), 7652-7657.

(3) Julich-Gruner, K. K.; Lowenberg, C.; Neffe, A. T.; Behl, M.; Lendlein, A. Recent Trends in the Chemistry of Shape-memory Polymers. Macromol. Chem. Phys. 2013, 214 (5), 527-536.

(4) Lendlein, A.; Jiang, H. Y.; Junger, O.; Langer, R. Light-Induced Shape-Memory Polymers. Nature 2005, 434 (7035), 879-882.

(5) Wang, S. Q.; Kaneko, D.; Okajima, M.; Yasaki, K.; Tateyama, S.; Kaneko, T. Hyperbranched Polycoumarates with Photofunctional Multiple Shape Memory. Angew. Chem., Int. Ed. 2013, 52 (42), $11143-11148$.

(6) Mohr, R.; Kratz, K.; Weigel, T.; Lucka-Gabor, M.; Moneke, M.; Lendlein, A. Initiation of Shape-memory Effect by Inductive Heating of Magnetic Nanoparticles in Thermoplastic Polymers. Proc. Natl. Acad. Sci. U. S. A. 2006, 103 (10), 3540-3545.

(7) Yakacki, C. M.; Shandas, R.; Lanning, C.; Rech, B.; Eckstein, A.; Gall, K. Unconstrained Recovery Characterization of Shape-memory Polymer Networks for Cardiovascular Applications. Biomaterials 2007, 28 (14), 2255-2263.

(8) Sharifi, S.; van Kooten, T. G.; Kranenburg, H. J. C.; Meij, B. P.; Behl, M.; Lendlein, A.; Grijpma, D. W. An Annulus Fibrosus Closure Device Based on a Biodegradable Shape-memory Polymer Network. Biomaterials 2013, 34 (33), 8105-8113.

(9) Lendlein, A.; Langer, R. Biodegradable, Elastic Shape-memory Polymers for Potential Biomedical Applications. Science 2002, 296 (5573), 1673-1676.

(10) Zhang, D. W.; George, O. J.; Petersen, K. M.; Jimenez-Vergara, A. C.; Hahn, M. S.; Grunlan, M. A. A Bioactive "Self-Fitting" Shape 
Memory Polymer Scaffold with Potential to Treat Cranio-maxillo

Facial Bone Defects. Acta Biomater. 2014, 10 (11), 4597-4605.

(11) Baker, R. M.; Tseng, L. F.; Iannolo, M. T.; Oest, M. E.; Henderson, J. H. Self-Deploying Shape Memory Polymer Scaffolds for Grafting and Stabilizing Complex Bone Defects: A Mouse Femoral Segmental Defect Study. Biomaterials 2016, 76, 388-398.

(12) Behl, M.; Lendlein, A. Triple-Shape Polymers. J. Mater. Chem. 2010, 20 (17), 3335-3345.

(13) Xie, T. Tunable Polymer Multi-Shape Memory Effect. Nature 2010, 464 (7286), 267-270.

(14) Huang, W. M.; Yang, B.; An, L.; Li, C.; Chan, Y. S. Water-driven Programmable Polyurethane Shape Memory Polymer: Demonstration and Mechanism. Appl. Phys. Lett. 2005, 86 (11), 114105.

(15) Kumpfer, J. R.; Rowan, S. J. Thermo-, Photo-, and ChemoResponsive Shape-memory Properties from Photo-Cross-Linked Metallo-supramolecular Polymers. J. Am. Chem. Soc. 2011, 133 (32), $12866-12874$

(16) Fang, Y.; Ni, Y. L.; Leo, S. Y.; Taylor, C.; Basile, V.; Jiang, P. Reconfigurable Photonic Crystals Enabled by Pressure-responsive Shape-Memory Polymers. Nat. Commun. 2015, 6, 7416.

(17) Jost, V.; Langowski, H.-C. Effect of Different Plasticisers on the Mechanical and Barrier Properties of Extruded Cast Phbv Films. Eur. Polym. J. 2015, 68, 302-312.

(18) Xiao, X. L.; Hu, J. L. Animal Hairs as Water-stimulated Shape Memory Materials: Mechanism and Structural Networks in Molecular Assemblies. Sci. Rep. 2016, 6, 26393.

(19) Xu, J. Q.; Bohnsack, D. A.; Mackay, M. E.; Wooley, K. L. Unusual Mechanical Performance of Amphiphilic Crosslinked Polymer Networks. J. Am. Chem. Soc. 2007, 129 (3), 506-507.

(20) Bedoui, F.; Widjaja, L. K.; Luk, A.; Bolikal, D.; Murthy, N. S.; Kohn, J. Anomalous Increase in Modulus Upon Hydration in Random Copolymers with Hydrophobic Segments and Hydrophilic Blocks. Soft Matter 2012, 8 (7), 2230-2236.

(21) Kutikov, A. B.; Song, J. An Amphiphilic Degradable Polymer/ Hydroxyapatite Composite with Enhanced Handling Characteristics Promotes Osteogenic Gene Expression in Bone Marrow Stromal Cells. Acta Biomater. 2013, 9 (9), 8354-8364.

(22) Zheng, J. F.; Liu, X.; Chen, X. F.; Ren, X. K.; Yang, S.; Chen, E. Q. Hemiphasmidic Side-Chain Liquid Crystalline Polymer: From Smectic C Phase to Columnar Phase with a Bundle of Chains as Its Building Block. ACS Macro Lett. 2012, 1 (5), 641-645.

(23) Glatter, O.; Kratky, O. Small Angle X-Ray Scattering; Academic Press: Orlando, FL, 1982.

(24) Chen, C.; Chen, L.; Cao, L.; Shen, W.; Yu, L.; Ding, J. Effects of $L$-Lactide and $D, L$-Lactide in Poly(lactide-co-glycolide)-poly(ethylene glycol)-poly(lactide-co-glycolide) on the Bulk States of Triblock Copolymers, and Their Thermogellation and Biodegradation in Water. RSC Adv. 2014, 4 (17), 8789-8798.

(25) Saliba, J. B.; da Silva-Cunha Junior, A.; da Silva, G. R.; Yoshida, M. I.; Piscitelli Mansur, A. A.; Mansur, H. S. Characterization and in Vitro Release of Cyclosporine-a from $\operatorname{Poly}(D, L$-lactide-co-glycolide Implants Obtained by Solvent/Extraction Evaporation. Quim. Nova 2012, 35 (4), 723-727.

(26) Ignjatovic, N.; Ninkov, P.; Ajdukovic, Z.; Vasiljevic-Radovic, D.; Uskokovic, D. Biphasic Calcium Phosphate Coated with Poly-D,Llactide-co-glycolide Biomaterial as a Bone Substitute. J. Eur. Ceram. Soc. 2007, 27 (2-3), 1589-1594.

(27) d'Acunzo, F.; Kohn, J. Alternating Multiblock Amphiphilic Copolymers of PEG and Tyrosine-Derived Diphenols. 1. Synthesis and Characterization. Macromolecules 2002, 35 (25), 9360-9365.

(28) Xu, J.; Bohnsack, D. A.; Mackay, M. E.; Wooley, K. L. Unusual Mechanical Performance of Amphiphilic Crosslinked Polymer Networks. J. Am. Chem. Soc. 2007, 129 (3), 506-507. 